\title{
THE TOPOLOGY OF AN OPEN MANIFOLD WITH RADIAL CURVATURE BOUNDED FROM BELOW BY A MODEL SURFACE WITH FINITE TOTAL CURVATURE AND EXAMPLES OF MODEL SURFACES
}

\author{
MINORU TANAKA AND KEI KONDO
}

\begin{abstract}
We construct distinctive surfaces of revolution with finite total curvature whose Gauss curvatures are not bounded. Such a surface of revolution is employed as a reference surface of comparison theorems in radial curvature geometry. Moreover, we prove that a complete noncompact Riemannian manifold $M$ is homeomorphic to the interior of a compact manifold with boundary if the manifold $M$ is not less curved than a noncompact model surface $\widetilde{M}$ of revolution and if the total curvature of the model surface $\widetilde{M}$ is finite and less than $2 \pi$. By the first result mentioned above, the second result covers a much wider class of manifolds than that of complete noncompact Riemannian manifolds whose sectional curvatures are bounded from below by a constant.
\end{abstract}

\section{$\S 1$. Introduction}

In a series of previous articles (see [KT1], [KT2], and [KT3]), by restricting the total curvature of a noncompact model surface of revolution we investigated some topological properties of a complete and noncompact Riemannian manifold which is not less curved than the model surface. (The precise definition of "not less curved than a noncompact model surface of revolution" is given later in this article.) Typical noncompact model surfaces are a Euclidean plane $\left(\mathbb{R}^{2}, d t^{2}+t^{2} d \theta^{2}\right)$ and a hyperbolic plane $\left(\mathbb{R}^{2}, d t^{2}+\sinh ^{2} t d \theta^{2}\right)$. Here $(t, \theta)$ denotes polar coordinates around the origin of $\mathbb{R}^{2}$. We construct a noncompact model surface of revolution $(\widetilde{M}, \tilde{p})$ as follows. Let a smooth function $f:(0, \infty) \longrightarrow(0, \infty)$ be given. Then, $\left(\mathbb{R}^{2}, d t^{2}+f(t)^{2} d \theta^{2}\right)$ is a noncompact complete surface of revolution $\widetilde{M}$ with smooth Riemannian metric $d t^{2}+f(t)^{2} d \theta^{2}$ around the base point $\tilde{p} \in \widetilde{M}$, if $f$ is extensible to a smooth odd function around 0 and satisfies $f^{\prime}(0)=1$ (see [SST, Theorem 7.1.1]). It is well known that the Gauss curvature $G$ of

Received February 22, 2011. Revised November 8, 2011. Accepted January 9, 2012. 2010 Mathematics Subject Classification. Primary 53C21; Secondary 53C22. 
$\widetilde{M}$ is given by

$$
G(q)=-\frac{f^{\prime \prime}}{f}(t(q)) .
$$

The total curvature $c(\widetilde{M})$ of a noncompact model surface of revolution $\widetilde{M}$ is defined by

$$
c(\widetilde{M}):=\int_{\widetilde{M}} G_{+} d \widetilde{M}+\int_{\widetilde{M}} G_{-} d \widetilde{M},
$$

if $\int_{\widetilde{M}} G_{+} d \widetilde{M}<\infty$ or $\int_{\widetilde{M}} G_{-} d \widetilde{M}>-\infty$. Here $G_{+}:=\max \{G, 0\}, G_{-}:=$ $\min \{G, 0\}$, and $d \widetilde{M}$ denotes the area element of $\widetilde{M}$. The total curvature of a complete 2-dimensional Riemannian manifold is defined analogously. This definition was introduced by Cohn-Vossen in [CV].

In 1935, Cohn-Vossen generalized the Gauss-Bonnet theorem for noncompact Riemannian manifolds, as follows.

TheOREM 1.1 ([CV, Satz 6]). If a connected, complete, noncompact, finitely connected Riemannian 2-dimensional manifold $X$ admits a total curvature $c(X)$, then

$$
c(X) \leq 2 \pi \chi(X)
$$

holds. Here $\chi(X)$ denotes the Euler characteristic of $X$.

Now, we are in a position to give the precise definition of "not less curved than a noncompact model surface of revolution" in the following. Let $(M, p)$ denote a complete, connected, and noncompact $n$-dimensional Riemannian manifold with base point $p \in M$ and $(\widetilde{M}, \tilde{p})$ a noncompact model surface of revolution defined above. Note that a unit speed geodesic $\widetilde{\gamma}:[0, \infty) \longrightarrow \widetilde{M}$ emanating from $\tilde{p}$, which is called a meridian, is a ray. From now on, we choose a meridian $\widetilde{\gamma}$ and fix it. We say that the manifold $(M, p)$ has radial curvature at the base point $p$ bounded from below by that of the model surface $(\widetilde{M}, \tilde{p})$ if, along every minimal geodesic $\gamma:[0, a) \longrightarrow M$ emanating from $p=\gamma(0)$, its sectional curvature $K_{M}$ satisfies

$$
K_{M}\left(\sigma_{t}\right) \geq G(\widetilde{\gamma}(t))
$$

for all $t \in[0, a)$ and for all 2-dimensional linear planes $\sigma_{t}$ containing $\gamma^{\prime}(t)$. This is the precise definition that a complete noncompact Riemannian manifold is not less curved than a model surface.

By Theorem 1.1, the total curvature of a noncompact model surface of revolution does not exceed $2 \pi$ if the total curvature exists. Hence, it is 
natural to assume that the total curvature of a noncompact model surface of revolution is finite. Under this assumption, we have proved the following theorem.

TheOREM 1.2 ([KT2, Theorem 2.2]). Let $(M, p)$ be a complete noncompact Riemannian manifold $M$ whose radial sectional curvature at the base point $p$ is bounded from below by that of a noncompact model surface of revolution $(\widetilde{M}, \tilde{p})$ with its metric $d t^{2}+f(t)^{2} d \theta^{2}$. If

(1) $\widetilde{M}$ admits a finite total curvature, and

(2) $\widetilde{M}$ has no pair of cut points in a sector $\widetilde{V}\left(\delta_{0}\right)$ for some $\delta_{0} \in(0, \pi]$,

then $M$ is homeomorphic to the interior of a compact manifold with boundary. Here $\widetilde{V}\left(\delta_{0}\right):=\left\{\tilde{x} \in \widetilde{M} \mid 0<\theta(\tilde{x})<\delta_{0}\right\}$.

In this article, we will show that item (2) of Theorem 1.2 is unnecessary if the total curvature is less than $2 \pi$. That is, we will prove the following theorem.

THEOREM 1.3. A connected, complete, noncompact Riemannian manifold $(M, p)$ is homeomorphic to the interior of a compact manifold with boundary if the radial curvature at a point $p \in M$ is bounded from below by that of a noncompact model surface of revolution $(\widetilde{M}, \tilde{p})$ which admits a finite total curvature $c(\widetilde{M})$ less than $2 \pi$.

Note that the finiteness of the total curvature does not impose strong restriction on the curvature of the model surface. In fact, we will prove the following theorem, which tells us that the radial curvature of the model surface in Theorem 1.3 is not always bounded from below.

THEOREM 1.4. Let $\widetilde{M}:=\left(\mathbb{R}^{2}, d t^{2}+f(t)^{2} d \theta^{2}\right)$ denote a noncompact model surface of revolution which admits a finite total curvature $c(\widetilde{M})$ less than $2 \pi$. Then, for any $\varepsilon>0$, there exists a noncompact model surface of revolution $\widetilde{M}_{\varepsilon}^{-}:=\left(\mathbb{R}^{2}, d t^{2}+m_{\varepsilon}^{-}(t) d \theta^{2}\right)$ such that

$$
K \geq G_{\varepsilon}^{-} \quad \text { on }[0, \infty), \quad\left\|G_{\varepsilon}^{-}-K\right\|_{2}<\varepsilon, \quad \liminf _{t \rightarrow \infty} G_{\varepsilon}^{-}(t)=-\infty,
$$

and

$$
\left|c(\widetilde{M})-c\left(\widetilde{M}_{\varepsilon}^{-}\right)\right|<\varepsilon
$$

Here the functions

$$
K(t):=-\frac{f^{\prime \prime}}{f}(t), \quad G_{\varepsilon}^{-}(t):=-\frac{m_{\varepsilon}^{-\prime \prime}}{m_{\varepsilon}^{-}}(t)
$$


denote the radial curvature of $\widetilde{M}, \widetilde{M}_{\varepsilon}^{-}$, respectively, and $\left\|G_{\varepsilon}^{-}-K\right\|_{2}:=$ $\sqrt{\int_{0}^{\infty}\left|G_{\varepsilon}^{-}-K\right|^{2} d t}$.

REMARK 1.5. In Theorem 1.4, it is impossible to choose $\widetilde{M}_{\varepsilon}^{-}$as a von Mangoldt surface of revolution, when $K(t)$ is bounded from below. Here a von Mangoldt surface of revolution is, by definition, a model surface of revolution whose radial curvature is nonincreasing on $[0, \infty)$.

\section{§2. Proof of Theorem 1.3}

By the same argument used in the proof of [KT2, Theorem 5.3], we have the following lemma.

LEMMA 2.1. Let $\left(M^{*}, p^{*}\right)$ be a noncompact model surface of revolution with its metric $d t^{2}+m(t)^{2} d \theta^{2}$ satisfying the differential equation $m^{\prime \prime}(t)+$ $K(t) m(t)=0$ with initial conditions $m(0)=0$ and $m^{\prime}(0)=1$. If $M^{*}$ satisfies

$$
\int_{0}^{\infty} t K(t) d t>-\infty
$$

and if $K(t) \leq 0$ on $[0, \infty)$, then $M^{*}$ admits a finite total curvature.

LEMma 2.2. Let $(\widetilde{M}, \tilde{p})$ denote a noncompact model surface of revolution with its metric $d \tilde{s}^{2}=d t^{2}+f(t)^{2} d \theta^{2}$ satisfying the differential equation $f^{\prime \prime}(t)+G(t) f(t)=0$ with initial conditions $f(0)=0$ and $f^{\prime}(0)=1$. If $\widetilde{M}$ admits a finite total curvature $c(\widetilde{M})$ less than $2 \pi$, then there exists a noncompact model surface of revolution $\left(M^{*}, p^{*}\right)$ with its metric

$$
g^{*}=d t^{2}+m(t)^{2} d \theta^{2}
$$

satisfying the differential equation $m^{\prime \prime}(t)+G_{-}(t) m(t)=0$ with initial conditions $m(0)=0$ and $m^{\prime}(0)=1$ such that $M^{*}$ admits a finite total curvature. Here $G_{-}:=\min \{G, 0\}$.

Proof. Since $\widetilde{M}$ admits a finite total curvature, it follows from [SST, (5.2.6)] that $\lim _{t \rightarrow \infty} f^{\prime}(t) \in \mathbb{R}$ exists, and it also follows from [SST, Theorem 5.2.1] that

$$
2 \pi \lim _{t \rightarrow \infty} f^{\prime}(t)=\lim _{t \rightarrow \infty} \frac{2 \pi f(t)}{t}=2 \pi-c(\widetilde{M})
$$

holds. Since $-\infty<c(\widetilde{M})<2 \pi$ and since

$$
\lim _{t \downarrow 0} \frac{f(t)}{t}=1,
$$


there exists a positive constant $\alpha$ such that

$$
\frac{f(t)}{t}>\frac{1}{\alpha}
$$

on $(0, \infty)$. Thus,

$$
\int_{0}^{\infty} t G_{-}(t) d t \geq \alpha \int_{0}^{\infty} f(t) G_{-}(t) d t .
$$

Since $c(\widetilde{M})$ is finite,

$$
-\infty<\int_{\widetilde{M}} G_{-} \circ t d \widetilde{M}=2 \pi \int_{0}^{\infty} f(t) G_{-}(t) d t .
$$

By (2.2) and (2.3),

$$
\int_{0}^{\infty} t G_{-}(t) d t>-\infty
$$

Therefore, by Lemma 2.1, we get the noncompact model surface of revolution $\left(M^{*}, p^{*}\right)$ with the metric $(2.1)$ whose total curvature is finite.

Proof of Theorem 1.3. By Lemma 2.2, we have a noncompact model surface of revolution $\left(M^{*}, p^{*}\right)$ with its metric $(2.1)$ whose total curvature is finite. Since $G \geq G_{-}=\min \{G, 0\},\left(M^{*}, p^{*}\right)$ is the reference surface to $(M, p)$. Moreover, $\left(M^{*}, p^{*}\right)$ has no pair of cut points in a sector $\widetilde{V}(\delta)$ for all $\delta \in(0, \pi]$, since $0 \geq G_{-}$. Therefore, by Theorem $1.2, M$ is homeomorphic to the interior of a compact manifold with boundary.

\section{$\S 3 . \quad$ Fundamental lemmas}

We need several lemmas for constructing a family of specific surfaces of revolution. Let $K:[0, \infty) \longrightarrow \mathbb{R}$ be a continuous function, and let $f:$ $[0, \infty) \longrightarrow \mathbb{R}$ be a solution of the differential equation

$$
f^{\prime \prime}(t)+K(t) f(t)=0 .
$$

Here we assume that the solution $f$ satisfies

$$
f>0
$$

on $(0, \infty)$ and that

$$
\int_{1}^{\infty} f(t)^{-2} d t<\infty
$$


Lemma 3.1. Let $G:[0, \infty) \longrightarrow \mathbb{R}$ be a continuous function, and let $m$ be the solution of the differential equation

$$
m^{\prime \prime}(t)+G(t) m(t)=0
$$

with initial conditions $m(0)=f(0)$ and $m^{\prime}(0)=f^{\prime}(0)$. If $G-K$ has a compact support in a bounded interval $[a, b] \subset[1, \infty)$, then, for any $t \geq a$,

$$
|\sigma(t)| \leq \int_{a}^{t} f(t)^{-2}\left|m^{\prime} f-m f^{\prime}\right| d t
$$

holds. Here we set

$$
\sigma(t):=\frac{m}{f}(t)-1
$$

Proof. Since

$$
\sigma^{\prime}(t)=\frac{1}{f^{2}}\left(m^{\prime} f-m f^{\prime}\right)(t)
$$

and since $\sigma(t)=0$ on $(0, a]$, we obtain

$$
\sigma(t)=\int_{a}^{t} \frac{1}{f^{2}}\left(m^{\prime} f-m f^{\prime}\right)(t) d t
$$

and hence

$$
|\sigma(t)| \leq \int_{a}^{t} \frac{1}{f^{2}}\left|m^{\prime} f-m f^{\prime}\right| d t
$$

LEMmA 3.2. If $G$ and $m$ are the functions defined in Lemma 3.1, then

$$
\left|\left(m^{\prime} f-m f^{\prime}\right)(t)\right| \leq(\alpha(m)+1) \cdot\|G-K\|_{2} \cdot\left\|\left.f^{2}\right|_{[a, b]}\right\|_{2}
$$

holds on $[0, \infty)$. Here we set

$$
\|G-K\|_{2}:=\sqrt{\int_{0}^{\infty}|(G-K)(t)|^{2} d t}, \quad\left\|\left.f^{2}\right|_{[a, b]}\right\|_{2}:=\sqrt{\int_{a}^{b} f(t)^{4} d t}
$$

and $\alpha(m):=\sup _{t \geq 0}|\sigma(t)|$.

Proof. Since the case where $t \in[0, a]$ is trivial, we assume that $t>a$. By (3.1) and (3.4), we have

$$
\left(f m^{\prime}-f^{\prime} m\right)^{\prime}(t)=(K-G) f m(t)
$$


Hence,

$$
\left(f m^{\prime}-f^{\prime} m\right)(t)=\left(f m^{\prime}-f^{\prime} m\right)(b)
$$

holds for any $t \geq b$, since $G=K$ on $[b, \infty)$. By $(3.7)$, we get

$$
\left|\left(f m^{\prime}-f^{\prime} m\right)\right|(t) \leq \int_{a}^{t}|K-G| f^{2}(|\sigma|+1) d t=(\alpha(m)+1) \int_{a}^{t}|K-G| f^{2} d t .
$$

Now, it is clear from the Schwarz inequality and from (3.8) that (3.6) holds for any $t \geq 0$.

Lemma 3.3. Set

$$
C(f, a, b):=\int_{a}^{\infty} \frac{1}{f^{2}} d t \cdot\left\|\left.f^{2}\right|_{[a, b]}\right\|_{2}(>0)
$$

If

$$
C(f, a, b)<\frac{1}{\|G-K\|_{2}}
$$

then

$$
\alpha(m) \leq \frac{C(f, a, b)\|G-K\|_{2}}{1-C(f, a, b)\|G-K\|_{2}}
$$

Proof. Since $\sigma(t)=0$ for any $t \in[0, a]$, it follows from (3.5) and (3.6) that

$$
\sup _{t \geq 0}|\sigma(t)| \leq C(f, a, b) \cdot\|G-K\|_{2}(\alpha(m)+1) .
$$

Thus, it is clear that (3.9) holds.

LEMma 3.4. The equations

$$
\int_{a}^{b}|G m-K f| d t \leq(\alpha(m)+1)\|G-K\|_{2} \cdot\left\|\left.f\right|_{[a, b]}\right\|_{2}+\alpha(m) \int_{a}^{b}\left|f^{\prime \prime}\right| d t
$$

and

$$
\int_{b}^{\infty}|G m-K f| d t \leq \alpha(m) \int_{b}^{\infty}\left|f^{\prime \prime}\right| d t
$$

hold. Hence, we get

$$
\begin{aligned}
& \int_{0}^{\infty}|G m-K f| d t \\
& \quad \leq \alpha(m) \int_{a}^{\infty}\left|f^{\prime \prime}\right| d t+(\alpha(m)+1)\|G-K\|_{2} \cdot\left\|\left.f\right|_{[a, b]}\right\|_{2}
\end{aligned}
$$


Proof. Since

$$
(G m-K f)(t)=(G-K)(t) f(t)(\sigma(t)+1)+K(t) f(t) \sigma(t),
$$

we get, by the triangle inequality,

$$
|G m-K f|(t) \leq(\alpha(m)+1)|G-K|(t) f(t)+\alpha(m)|K(t) f(t)| .
$$

From the Schwarz inequality, it follows that

$$
\begin{aligned}
& \int_{a}^{b}|G-K|(t) f(t) d t \\
& \quad \leq(\alpha(m)+1)\|G-K\|_{2} \cdot\left\|\left.f\right|_{[a, b]}\right\|_{2}+\alpha(m) \int_{a}^{b}|K f| d t .
\end{aligned}
$$

Equation (3.10) is clear from (3.15), since $K f=-f^{\prime \prime}$ by (3.1). Since $\operatorname{supp}(G-K) \subset[a, b], G=K$ on $[b, \infty)$. Hence, $|G m-K f|(t)=|K f \sigma(t)| \leq$ $\alpha(m)|K f|(t)$ on $[b, \infty)$, and $G m(t)=K f(t)$ on $[0, a]$. Now, (3.11) and (3.12) are clear.

LEMmA 3.5. If $\alpha(m)<1$, then $m(t)>0$ on $(0, \infty)$, and

$$
\int_{1}^{\infty}\left|f(t)^{-2}-m(t)^{-2}\right| d t \leq \frac{(2+\alpha(m)) \alpha(m)}{(1-\alpha(m))^{2}} \int_{a}^{\infty} f(t)^{-2} d t
$$

Proof. Since $\sigma(t) \geq-\sigma(m)>-1$ for any $t \in[0, \infty)$, it is clear that $m(t)$ is positive on $(0, \infty)$. By definition, $m(t)^{-2}=(\sigma+1)^{-2} f(t)^{-2}$ holds. Hence, we get

$$
\left|f(t)^{-2}-m(t)^{-2}\right|=f(t)^{-2}\left|(\sigma+1)^{-2}-1\right| \leq \alpha(m) \cdot f(t)^{-2} \frac{|\sigma(t)|+2}{(1-|\sigma(t)|)^{2}} .
$$

Since the function $(x+2) /(1-x)^{2}$ is increasing on $[0,1)$,

$$
\left|f(t)^{-2}-m(t)^{-2}\right| \leq \frac{\alpha(m)(2+\alpha(m))}{(1-\alpha(m))^{2}} f(t)^{-2} .
$$

Since $G=K$ on $[0, a], f=m$ on $[0, a]$. Therefore, by (3.17),

$$
\begin{aligned}
\int_{1}^{\infty}\left|f(t)^{-2}-m(t)^{-2}\right| d t & =\int_{a}^{\infty}\left|f(t)^{-2}-m(t)^{-2}\right| d t \\
& \leq \frac{\alpha(m)(2+\alpha(m))}{(1-\alpha(m))^{2}} \int_{a}^{\infty} f(t)^{-2} d t
\end{aligned}
$$


Proposition 3.6. Let $K:[0, \infty) \longrightarrow \mathbb{R}$ be a continuous function, and let $f:[0, \infty) \longrightarrow \mathbb{R}$ be the solution of the differential equation of (3.1) with initial conditions $f(0)=0$ and $f^{\prime}(0)=1$. Suppose that the solution $f$ satisfies (3.2), (3.3), and

$$
\int_{0}^{\infty}\left|f^{\prime \prime}(t)\right| d t<\infty
$$

Then, for any $\varepsilon>0$ and for any bounded interval $(a, b) \subset[1, \infty)$, there exists $\delta>0$ such that, for any continuous function $G:[0, \infty) \longrightarrow \mathbb{R}$ satisfying $\operatorname{supp}(G-K) \subset[a, b]$ and

$$
\|G-K\|_{2}:=\sqrt{\int_{0}^{\infty}|G-K|^{2} d t}<\delta,
$$

the solution $m$ of the differential equation $m^{\prime \prime}(t)+G(t) m(t)=0$ with initial conditions $m(0)=0$ and $m^{\prime}(0)=1$ satisfies

$$
\int_{0}^{\infty}|G m(t)-K f(t)| d t<\varepsilon
$$

and

$$
\int_{1}^{\infty}\left|m(t)^{-2}-f(t)^{-2}\right| d t<\varepsilon
$$

Proof. Let $\varepsilon$ be an arbitrarily fixed number. Here we choose a positive number $\delta_{1} \in(0,1 / C(f, a, b))$ such that

$$
\frac{\delta_{1}}{1-C(f, a, b) \delta_{1}}\left\|\left.f\right|_{[a, b]}\right\|_{2}<\frac{\varepsilon}{2}
$$

and

$$
\frac{C(f, a, b) \delta_{1}}{1-C(f, a, b) \delta_{1}} \int_{a}^{\infty}\left|f^{\prime \prime}\right| d t<\frac{\varepsilon}{2}
$$

hold. Then it follows from Lemma 3.3, (3.20), and (3.21) that, for any continuous function $G:[0, \infty) \longrightarrow \mathbb{R}$ satisfying $\operatorname{supp}(G-K) \subset[a, b]$ and $\|G-K\|_{2}<\delta_{1}$, the solution $m$ satisfies

$$
\alpha(m) \int_{a}^{\infty}\left|f^{\prime \prime}\right| d t<\frac{\varepsilon}{2}
$$


and

$$
(\alpha(m)+1)\|G-K\|_{2} \cdot\left\|\left.f\right|_{[a, b]}\right\|_{2}<\frac{\varepsilon}{2} .
$$

Now, (3.18) is clear from (3.12). Moreover, by (3.16) and (3.9), there exists $\delta \in\left(0, \delta_{1}\right]$ such that, for any continuous function $G:[0, \infty) \longrightarrow \mathbb{R}$ satisfying $\operatorname{supp}(G-K) \subset[a, b]$ and $\|G-K\|_{2}<\delta<\delta_{1}$, the solution $m$ satisfies (3.18) and (3.19).

The following proposition is clear from Lemmas 3.1, 3.2, 3.3, and 3.5 and the proof of Proposition 3.6.

Proposition 3.7. Let $K:[0, \infty) \longrightarrow \mathbb{R}$ be a continuous function, and let $f:[0, \infty) \longrightarrow \mathbb{R}$ be the solution of the differential equation of (3.1) with initial conditions $f(0)=0$ and $f^{\prime}(0)=1$. Suppose that the solution $f$ satisfies (3.2) and (3.3). Then for any $\varepsilon>0$ and for any bounded interval $(a, b) \subset[1, \infty)$ there exists $\delta>0$ such that, for any continuous function $G:[0, \infty) \longrightarrow \mathbb{R}$ satisfying $\operatorname{supp}(G-K) \subset[a, b]$ and

$$
\|G-K\|_{2}:=\sqrt{\int_{0}^{\infty}|G-K|^{2} d t}<\delta,
$$

the solution $m$ of the differential equation $m^{\prime \prime}(t)+G(t) m(t)=0$ with initial conditions $m(0)=0$ and $m^{\prime}(0)=1$ satisfies (3.19).

\section{$\S 4$. The construction of a distinctive model}

Proof of Theorem 1.4. From the isoperimetric inequalities (see [SST, Theorem 5.2.1]) and l'Hôpital's theorem, it follows that

$$
2 \pi \lim _{t \rightarrow \infty} f^{\prime}(t)=\lim _{t \rightarrow \infty} \frac{2 \pi f(t)}{t}=2 \pi-c(\widetilde{M}) .
$$

Hence, the property $c(\widetilde{M})<2 \pi$ implies that

$$
\lim _{t \rightarrow \infty} \frac{f(t)}{t}=\lim _{t \rightarrow \infty} f^{\prime}(t)>0
$$

In particular,

$$
\int_{1}^{\infty} f(t)^{-2} d t<\infty
$$

Since $c(\widetilde{M})$ is finite,

$$
2 \pi \int_{0}^{\infty}|K(t)| f(t) d t<\infty
$$


This is equivalent to

$$
\int_{0}^{\infty}\left|f^{\prime \prime}(t)\right| d t<\infty
$$

By applying Proposition 3.6 for the interval $(3 / 2,5 / 2)$ and $\varepsilon / 9 \pi$, we may find a smooth function $G_{1}:[0, \infty) \longrightarrow \mathbb{R}$ such that $\left\|G_{1}-K\right\|_{2}<\varepsilon / 3^{2}, K \geq G_{1}$ on $[0, \infty), \operatorname{supp}\left(K-G_{1}\right) \subset(3 / 2,5 / 2)$,

$$
\int_{0}^{\infty}\left|m_{1}^{\prime \prime}-f^{\prime \prime}\right| d t<\frac{\varepsilon}{9 \pi}, \quad \int_{1}^{\infty}\left|m_{1}^{-2}-f^{-2}\right| d t<\frac{\varepsilon}{9 \pi}<\frac{\varepsilon}{9},
$$

and $\min \left\{G_{1}(t) ; 3 / 2 \leq t \leq 5 / 2\right\} \leq-1$. Here $m_{1}$ denotes the solution $m_{1}^{\prime \prime}+$ $G_{1} m_{1}=0$ with initial conditions $m_{1}(0)=0$ and $m_{1}^{\prime}(0)=1$. By applying Proposition 3.6, it is easy to define a sequence of smooth functions $\left\{G_{k}\right.$ : $[0, \infty) \longrightarrow \mathbb{R}\}_{k \geq 0}$, where $G_{0}=K$, satisfying $\left\|G_{k}-G_{k-1}\right\|_{2}<\varepsilon / 3^{k+1}, G_{k-1} \geq$ $G_{k}$ on $[0, \infty), \operatorname{supp}\left(G_{k}-G_{k-1}\right) \subset(2 k-1 / 2,2 k+1 / 2)$,

$$
\int_{0}^{\infty}\left|m_{k}^{\prime \prime}-m_{k-1}^{\prime \prime}\right| d t<\frac{\varepsilon}{3^{k+1} \pi}, \quad \int_{1}^{\infty}\left|m_{k}^{-2}-m_{k-1}^{-2}\right| d t<\frac{\varepsilon}{3^{k+1}},
$$

and $\min \left\{G_{k}(t) ; 2 k-1 / 2 \leq t \leq 2 k+1 / 2\right\} \leq-k$. Here $m_{k}$ denotes the solution of $m_{k}^{\prime \prime}+G_{k} m_{k}=0$ with initial conditions $m_{k}(0)=0$ and $m_{k}^{\prime}(0)=1$. We define $m_{\varepsilon}(t):=\lim _{k \rightarrow \infty} m_{k}(t)$ and $G_{\varepsilon}(t):=\lim _{k \rightarrow \infty} G_{k}(t)$. It is easy to check that $m_{\varepsilon}(t)$ is the solution of $m_{\varepsilon}{ }^{\prime \prime}+G_{\varepsilon}(t) m_{\varepsilon}(t)=0$ with initial conditions $m_{\varepsilon}(0)=0$ and $m_{\varepsilon}{ }^{\prime}(0)=1$. Furthermore, the function $m_{\varepsilon}$ and $G_{\varepsilon}$ satisfy

$$
\int_{0}^{\infty}\left|m_{\varepsilon}^{\prime \prime}-f^{\prime \prime}\right| d t \leq \frac{\varepsilon}{3 \pi}
$$

$\liminf _{t \rightarrow \infty} G_{\varepsilon}(t)=-\infty, K \geq G_{\varepsilon}$ on $[0, \infty)$, and $\left\|G_{\varepsilon}-K\right\|_{2} \leq \varepsilon / 3<\varepsilon$. Equation (4.1) implies that

$$
\left|c\left(\widetilde{M}_{\varepsilon}^{-}\right)-c(\widetilde{M})\right| \leq 2 \pi \int_{0}^{\infty}\left|m_{\varepsilon}^{\prime \prime}-f^{\prime \prime}\right| d t \leq \frac{2 \varepsilon}{3}<\varepsilon,
$$

where $\widetilde{M}_{\varepsilon}^{-}$is a noncompact model surface of revolution such that $\widetilde{M}_{\varepsilon}^{-}:=$ $\left(\mathbb{R}^{2}, d t^{2}+m_{\varepsilon}^{-}(t) d \theta^{2}\right)$ and $m_{\varepsilon}^{-}(t):=m_{\varepsilon}(t)$.

The proof of the following theorem is similar to the proof of Theorem 1.4 above.

THEOREM 4.1. Let $\widetilde{M}:=\left(\mathbb{R}^{2}, d t^{2}+f(t)^{2} d \theta^{2}\right)$ denote a noncompact model surface of revolution which admits a finite total curvature $c(\widetilde{M})$ less than $2 \pi$. Then, for any $\varepsilon>0$, there exists a noncompact model surface of revolution 
$\widetilde{M}_{\varepsilon}^{+}:=\left(\mathbb{R}^{2}, d t^{2}+m_{\varepsilon}^{+}(t) d \theta^{2}\right)$ such that $G_{\varepsilon}^{+} \geq K$ on $[0, \infty),\left\|G_{\varepsilon}^{+}-K\right\|_{2}<\varepsilon$, $\limsup _{t \rightarrow \infty} G_{\varepsilon}^{+}(t)=\infty$, and $\left|c(\widetilde{M})-c\left(M_{\varepsilon}^{+}\right)\right|<\varepsilon$, where we denote by $K:=$ $-f^{\prime \prime} / f, G_{\varepsilon}^{+}:=-m_{\varepsilon}^{+^{\prime \prime}} / m_{\varepsilon}+$ the radial curvature of $\widetilde{M}, \widetilde{M}_{\varepsilon}^{+}$, respectively.

Corollary 4.2. Let $\widetilde{M}:=\left(\mathbb{R}^{2}, d t^{2}+f(t)^{2} d \theta^{2}\right)$ denote a noncompact model surface of revolution which satisfies (3.2) and (3.3). Then, for any $\varepsilon>0$, there exist noncompact model surfaces of revolution $\widetilde{M}_{\varepsilon}^{+}:=\left(\mathbb{R}^{2}, d t^{2}+\right.$ $\left.m_{\varepsilon}^{+}(t) d \theta^{2}\right)$ and $\widetilde{M}_{\varepsilon}^{-}:=\left(\mathbb{R}^{2}, d t^{2}+m_{\varepsilon}^{-}(t) d \theta^{2}\right)$ such that $G_{\varepsilon}^{+} \geq K \geq G_{\varepsilon}^{-}$on $[0, \infty),\left\|G_{\varepsilon}^{*}-K\right\|_{2}<\varepsilon$, and $\int_{1}^{\infty}\left|f(t)^{-2}-m_{\varepsilon}^{*-2}\right| d t<\varepsilon$. Here $*= \pm 1$.

\section{REFERENCES}

[CV] S. Cohn-Vossen, Totalkrümmung und geodätische Linien auf einfachzusammenhängenden offenen vollständigen Flächenstücken, Rec. Math. Moscou (2) 1 (1936), 139-163. Zbl 0014.27601.

[KT1] K. Kondo and M. Tanaka, Total curvatures of model surfaces control topology of complete open manifolds with radial curvature bounded below, II, Trans. Amer. Math. Soc. 362 (2010), 6293-6324. MR 2678975. DOI 10.1090/ S0002-9947-2010-05031-7.

[KT2] - Total curvatures of model surfaces control topology of complete open manifolds with radial curvature bounded below, I, Math. Ann. 351 (2011), 251-266. MR 2836657. DOI 10.1007/s00208-010-0593-4.

[KT3] - Total curvatures of model surfaces control topology of complete open manifolds with radial curvature bounded below, III, J. Math. Soc. Japan 64 (2012), 185-200. MR 2879741. DOI 10.2969/jmsj/06410185.

[SST] K. Shiohama, T. Shioya, and M. Tanaka, The Geometry of Total Curvature on Complete Open Surfaces, Cambridge Tracts in Math. 159, Cambridge University Press, Cambridge, 2003. MR 2028047. DOI 10.1017/CBO9780511543159.

Minoru Tanaka

Department of Mathematics

Tokai University

Hiratsuka City, Kanagawa Pref.

259 - 1292 Japan

tanaka@tokai-u.jp

Kei Kondo

Department of Mathematics

Tokai University

Hiratsuka City, Kanagawa Pref.

259 - 1292 Japan

keikondo@keyaki.cc.u-tokai.ac.jp 